\title{
La desigualdad de ingreso y salud en Colombia
}

\author{
Sandra Rodríguez Acosta*
}

Perfiles Latinoamericanos, 24(48)

2016 | pp. 265-296

DOI: $10.18504 / \mathrm{pl} 2448-011-2016$

\section{Resumen}

Este trabajo plantea dos propósitos, el primero verificar si las condiciones económicas contextuales influyen en la salud autopercibida, independiente del estatus socioeconómico del individuo. Y segundo, explorar los mecanismos que operarían en el nivel de las regiones y que podrían explicar la influencia de las condiciones económicas estructurales, sobre la percepción de una salud pobre. Para esto se utiliza una metodología de análisis multinivel que permite separar los factores individuales y contextuales que influyen sobre la percepción de salud. Entre los resultados, se muestra que el estatus socioeconómico es un determinante fundamental de la percepción de salud de la población. No obstante, permanecen diferencias significativas en la salud percibida, aun después de controlar por la riqueza individual, el nivel educativo y la pertenencia étnica. Estas solo desaparecen cuando se tiene en cuenta la desigualdad de ingreso.

\begin{abstract}
The purpose of this paper is twofold: first, to examine independent of the individual socioeconomic status, the effect of the contextual economic conditions on the self-rated health and, second, to explore region-level mechanisms that could explain the influence of the structural economic conditions on the low self-rated health status. For this, we use a multilevel analysis that allows us to distinguish between the individual and the contextual factors influencing the self-rated health. The results show that the socioeconomic status is an important determinant of the self-rated health of population in Colombia. However, significant differences remain in the health perception, even after controlling for individual wealth, education level and ethnicity. These differences only disappear when controlling for income inequality in the analysis.
\end{abstract}

Palabras clave: salud autopercibida, desigualdad de ingreso, determinantes de salud, análisis multinivel, estatus socioeconómico.

Keywords: Self-rated health, income inequality, health determinants, multilevel analysis, socioeconomic status.

* PhD en Economía Aplicada de la Universidad Autónoma de Barcelona. Docente investigadora del Instituto de Estudios Económicos del Caribe (IEEC), Departamento de Economía, Universidad del Norte. 


\section{Introducción ${ }^{1}$}

L

Comisión sobre Determinantes Sociales de la Salud de la Organización Mundial de la Salud (oms), en su investigación sobre las causas sociales de la desigualdad en salud (OMS, 2011) concluye que esa desigualdad no puede explicarse únicamente por niveles de pobreza, variaciones en el ingreso o diferencias de capacidad de los servicios de salud locales, sino que adicionalmente se la puede explicar por la distribución desigual de condiciones sociales, políticas y económicas, a las que la oms ha denominado determinantes sociales de la salud. Y se enfatiza que las principales causas intermedias de la morbilidad y la mortalidad en los países de ingreso medio y alto se vinculan con la posición socioeconómica del individuo; es decir, las causas principales de las inequidades en salud surgen de las condiciones en las que nace, vive, trabaja y envejece la población (oms, 2011).

Contoyannis \& Foster (1999), aportando una visión económica a la relación entre ingreso y salud, proponen que los efectos sobre esta última, debido a un cambio o porcentaje de cambio en el ingreso, quizá no serían los mismos para todos los grupos sociales; y plantean que la relación suele tener influencia de factores externos. La desigualdad en los niveles de logro en salud y educación se deberían, más allá de la oferta de servicios existentes, a otras restricciones que operan en el ámbito doméstico, entre las que destacan las limitaciones que representan la desigualdad en los ingresos o las distintas situaciones que definen las tareas de la mujer en el hogar (PNUD, 2010).

Dichos factores son denominados por la oms determinantes intermedios de la salud. Es el caso de los estilos de vida, las condiciones de trabajo, la vivienda, las redes sociales, la educación, la recreación, etc., que son afectados en mayor o menor grado por el ingreso.

Este trabajo centra su objetivo en la preocupación de la oms en cuanto a identificar los determinantes sociales de las desigualdades en salud en Colombia; asimismo busca aportar a la discusión aún no concluyente sobre la relación desigualdad de ingreso-salud. Específicamente, se busca verificar, a la luz de esta hipótesis, la existencia de determinantes contextuales para la salud individual, medida a partir de la salud autopercibida. Son dos los propósitos: 1) verificar si las condiciones económicas contextuales inciden o influyen sobre la salud auto-

1 Esta investigación fue posible gracias al soporte financiero de la Universidad del Norte y el Departamento Administrativo de Ciencia y Tecnología (Colciencias) en Colombia. Se agradecen los importantes comentarios de Francesc Trillas (Universidad Autónoma de Barcelona), Joan Rovira (Universidad de Barcelona), Anna Matas (Universidad Autónoma de Barcelona) y Jaume Puig-Junoy (Universidad Pompeu Fabra). 
percibida, independientemente del estatus socioeconómico en el que se ubique el individuo, y 2) explorar los mecanismos que operarían en los niveles regional e individual, los cuales ayudarían a explicar la influencia de las condiciones económicas estructurales sobre la percepción de una salud pobre.

Para ello se utiliza la técnica de modelación multinivel, con la cual se verificaría la influencia de factores contextuales sobre la salud autopercibida. Específicamente, este análisis permite separar los efectos del ingreso individual, de los efectos contextuales de la desigualdad de ingreso.

Entre los resultados de la modelación, se observa que existen diferencias significativas en la percepción individual del estado de salud en muchas de las regiones de Colombia. Además, se encuentran evidencias de que los principales vínculos que relacionan el estado de salud con la desigualdad parecen ser el sentimiento de seguridad de los residentes en la región, así como la dotación de espacio público.

Este artículo se divide en cinco apartados. El primero corresponde a esta introducción. En el segundo se presenta la revisión de la literatura y el marco conceptual. En el tercero se describe el contexto del estudio y se explica la estrategia de modelación. En el cuarto se muestran los resultados del análisis multinivel para los determinantes de la salud autopercibida y, finalmente, en el último acápite se presentan las conclusiones.

\section{Revisión de la literatura y marco conceptual}

La evidencia sobre desigualdades relacionadas con el ingreso en la salud es ampliamente conocida (Kakwani et al. 1997; Doorslaer et al., 1997; Humphries $\&$ Doorslaer, 2000) y se sugiere que la salud individual es función del ingreso individual. Desde la sociología y la epidemiología hay un creciente soporte para la hipótesis, el cual establece que en países desarrollados la desigualdad de ingreso tiene un mayor impacto sobre la salud individual que el ingreso absoluto (Wilkinson, 1996).

\section{El debate teórico sobre la asociación salud-ingreso}

Muchos estudios han mostrado una asociación positiva entre ingreso y supervivencia (Viscusi, 1994; Lutter \& Morrall, 1994). Esto es consistente con la visión de que los incrementos en el ingreso acrecientan la inversión en bienes que mejoran la salud (Grossman, 2000). Esto último se refiere en la literatura como la hipótesis de ingreso absoluto (HIA), según la cual el ingreso absoluto y 
las condiciones materiales impactan la salud. Paralelo a esto, Wilkinson (1997, 1998) desarrolla una explicación alternativa para la desigualdad en salud, basada en el efecto que la desigualdad de ingreso ejerce sobre el estado de salud individual, denominada la hipótesis de ingreso relativo (HIR). Un bajo ingreso relativo podría, por ejemplo, estar asociado con incrementos en el estrés psicosocial generador de enfermedades (Cohen et al., 1991, 1997).

Aunque la evidencia ofrecida por los estudios longitudinales parece soportar la HIA (Gerdtham \& Johannesson, 2004), ambas explicaciones no son mutuamente excluyentes. Estas sugieren que reducir las desigualdades en salud sería importante para diseñar intervenciones que aborden lo psicosocial conjuntamente con los determinantes puramente materiales de la salud.

En combinación con la HIR, hay una tercera hipótesis que sugiere que la desigualdad de ingresos en sí sería un riesgo para la salud (Wilkinson, 1996). Esta hipótesis de desigualdad de ingresos-salud (HDI) ha sido soportada en comparaciones internacionales que muestran fuerte correlación entre desigualdad de ingreso y mortalidad, después de controlar por ingreso medio (Rodgers, 1979; Flegg, 1982; Waldmann, 1992; Wilkinson, 1996).

No obstante, como señala Smith (1999), es difícil hacer distinciones empíricas entre el efecto del ingreso y el efecto de la desigualdad de ingresos usando datos agregados. Mullahy et al. (2001) señalan que la evidencia de la relación entre ingreso individual y salud es alta y relativamente consistente; mientras que la evidencia para la relación entre medidas agregadas de desigualdad y salud sigue siendo débil y controversial. Por lo que, para diferenciar entre las distintas hipótesis, deberían usarse datos en un nivel individual.

A la misma conclusión llegan Wagstaff \& Doorslaer (2000), quienes clasifican la literatura que busca probar la relación distribución de ingreso-salud. Ellos concluyen que los estudios a nivel agregado son insuficientes para discriminar entre las hipótesis que vinculan la desigualdad de ingreso y la salud (Wilkinson, 1992; Anand \& Ravallion, 1993; Carrin \& Polti, 1995; Bidani \& Ravallion, 1997), y que en virtualmente todos los estudios a nivel de comunidades encuentran que la desigualdad de ingreso regional influye en la tasa de mortalidad, luego de controlar por nivel de ingresos (Kaplan et al., 1996; Kawachi et al., 1997; Kennedy et al., 1996).

La revisión de Wilkinson \& Pickett (2006) identifica 168 análisis en 155 trabajos de distintas disciplinas que reportan alguna asociación entre distribución de ingreso y salud de la población en países desarrollados. Y muestra que el $70 \%$ de los trabajos sugiere que el estado de salud es peor en sociedades donde las diferencias de ingreso son mayores. El centro de las investigaciones han sido los países desarrollados y son casi nulos los análisis sobre este fenómeno en América Latina, particularmente en Colombia. 
Es razonable considerar que el ingreso propio es un determinante de importancia en la salud individual. No obstante, hay otros mecanismos indirectos que la pueden determinar (Deaton, 2003). Este vínculo indirecto se debería a la desigualdad de ingreso, privación relativa, capital social u otras sendas de difícil conceptualización y medición. La evidencia de mala salud generada por jerarquías sociales es común en epidemiología y biología (Marmot et al., 1978; Sapolski, 1993).

La cuestión de que si la desigualdad del ingreso se relaciona o no con la salud y con la desigualdad en salud ha merecido atención en disciplinas como la economía (Gravelle, 1998; Contoyannis \& Forster, 1999; Contoyannis et al., 2004; Daly et al., 1998; Wildman, 2001, 2003), la sociología (Wilkinson, 1996; Wilkinson \& Pickett, 2009; Subramanian et al., 2002) y la epidemiología (Davey-Smith, 1996; Kawachi et al., 1999). Lo mismo ha ocurrido con el debate sobre la naturaleza del vínculo entre ingreso, desigualdad de ingreso, salud y desigualdad en salud. El más reciente y debatido trabajo en esta cuestión lo aportan Wilkinson \& Pickett (2009), cuya hipótesis es que una vez que los países han alcanzado cierto nivel de riqueza, lo que afecta a los ciudadanos no es el crecimiento del piB, sino el nivel de desigualdad. ${ }^{2}$

\section{Factores que influyen en el gradiente socioeconómico}

En economía, la influencia del estatus socioeconómico sobre la salud se debate especialmente a partir de las relaciones salud-educación (Grossman, 2000; Smith, 2004) y salud-ingreso (o riqueza) (Deaton, 2003; Smith, 2004). Normalmente los estudios económicos se enfocan en las medidas de ingreso, pero fallan al considerar la clasificación de la posición socioeconómica, o en controlar los efectos del conocimiento y los ambientales (Costa \& HernándezQuevedo, 2012).

Una forma de agrupar estos factores que influyen en el gradiente socioeconómico la presentan Lynch et al. (2000) en tres hipótesis explicativas: la interpretación del ingreso-individual, la interpretación del ambiente psicosocial y la interpretación neomaterialista. La primera se ha contrastado empíricamente

2 Han sido variadas las aproximaciones, principalmente en países desarrollados, pero los resultados siguen sujetos a controversia. Lo interesante de este debate es que ha llevado la desigualdad de ingreso y sus efectos sobre la salud a las agendas políticas en los países desarrollados y en América Latina. 
cuando, al controlar conforme al ingreso individual, permanece la asociación significativa entre desigualdad de ingreso y salud algunas veces, incluso después de controlar otras variables de estatus socioeconómico. ${ }^{3}$ Según Bowles $\&$ Gintis (2002), la riqueza, raza y escolaridad son importantes para la herencia del estatus económico.

Las aplicaciones económicas en esta perspectiva muestran que el ingreso de los padres y la riqueza tiene una fuerte asociación con la salud de los adultos, tanto para las familias en países en desarrollo (Poel \& Hosseinpoor, 2008), como en los desarrollados (Case et al., 2005). Jones \& Wildman (2008) encontraron, por su parte, un efecto significativo del ingreso corriente sobre la salud después de controlar por educación.

La interpretación psicosocial considera que la desigualdad per se produce un ambiente social que termina por perjudicar la salud. Wilkinson (1996) argumenta que las personas, al juzgar su nivel de vida comparándose con otros a su alrededor, son más conscientes de su bajo estatus social. Esto crea estrés, que a su vez se asocia a mayor incidencia de enfermedad cardiovascular, depresión, ansiedad, etc. La evidencia muestra que estos factores influyen en la salud y que su prevalencia está afectada por la estructura socioeconómica y por la posición que dentro de esta ocupa la persona. ${ }^{4}$

Por último, la interpretación neomaterialista considera que el mayor énfasis debe darse sobre los factores externos, o precursores de una vía psicosocial. Según esta visión, son los procesos estructurales, políticos y económicos los que generan la desigualdad, procesos que existen antes de que el individuo experimente o perciba sus efectos (Kaplan et al., 1996; Lynch et al., 1998; DaveySmith, 1996). A medida que las inversiones públicas (atención de la salud, seguridad pública, educación, calidad ambiental) estén concentradas en áreas de altos ingresos, o en áreas menos desiguales, entonces, el estándar de vida en esas áreas generará más acceso a esos bienes y servicios. Desde esta concepción, mayores desigualdades de ingreso en una sociedad conducirán a diferencias en los recursos a través de las comunidades, lo cual estará asociado con mayores diferencias en salud.

3 Esta relación se ha confirmado en Waitzman \& Smith (1998b), Daly et al. (1998), Soobader \& LeClere (1999), Kennedy et al. (1998), Fiscella \& Franks (2000). Y se ha rechazado en los de Fiscella \& Franks (1997), Mellor \& Milyo (1999) y LeClere \& Soobader (2000).

4 Kawachi et al. (1997) mostraron que la mayor igualdad se asocia con un mayor bienestar y pertenencia a grupos; mientras que la mayor desigualdad se vincula con la hostilidad. La epidemiología y la sociología sugieren que personas con más contacto social y más implicadas en actividades locales muestran mejor salud, incluso después de controlar otros factores. La evidencia epidemiológica ha demostrado que varias formas de estrés psicosocial tienen una influencia poderosa sobre las tasas de mortalidad y de enfermedad. 


\section{Contexto, metodología y datos}

Colombia, según el informe "Panorama social de América Latina 2013", publicado por la CEPAL $(2009,2013)$, aparece como uno de los países con la peor distribución del ingreso en la región — de acuerdo al indicador de diferencia entre los ingresos del 10\% más rico de la población y el $40 \%$ más pobre-; y en uno de los primeros lugares si se considera la relación clásica entre los quintiles I y V de ingresos. Si bien se ha reconocido una mejora en los niveles de equidad en Latinoamérica, en Colombia la situación ha sido más lenta que la tendencia regional. En 2009, Colombia pasó a ser el país más inequitativo de Sudamérica, superando a Brasil y Bolivia (pNud, 1999-2009). El índice de Gini en 2012 sigue ubicando a Colombia entre los más desiguales del continente.

Recientes estudios en Colombia han tratado de explorar el impacto que la reforma del sistema de salud, en 1993, tuvo sobre la equidad en salud y el uso de servicios (Castaño et al., 2000; Flórez \& Nupia, 2001; Céspedes et al., 2001; CIE Universidad de Antioquia, 2000; Florez \& Tono, 2002). Entre las investigaciones que examinan más que descriptivamente el fenómeno de la salud y la desigualdad en Colombia, están los de Bernal \& Cárdenas (2005) y Ruiz (2011). El primero observa que la mayoría de las disparidades raciales y étnicas en salud y acceso a esta desaparecen una vez que se controla por características socioeconómicas de los individuos, estatus de empleo y características del trabajo y ubicación geográfica, entre otras. Y Ruiz (2011) encuentra inequidad en la cobertura del seguro de salud, la autopercepción de una salud pobre y el acceso a los servicios médicos. También ilustran una distribución desigual en las condiciones de enfermedades crónicas entre diferentes grupos de población.

No obstante los avances en la literatura sobre desigualdad en salud, no se encuentran trabajos que examinen el problema desde la perspectiva de la hipótesis desigualdad de ingresos-salud, tampoco que traten de distinguir directamente entre los factores contextuales o individuales que subyacen en esta relación; de ahí que este trabajo pretenda aportar en esa línea.

\section{Modelo empírico: aproximación multinivel}

La distinción entre los efectos de privación absoluta (pobreza) de los efectos de privación relativa (desigualdad de ingreso) es difícil, porque la correlación entre desigualdad de ingreso y mortalidad de la población solo reflejaría los efectos de la pobreza sobre los excesos de mortalidad, y el hecho de que la alta concentración de individuos pobres se encuentra en áreas (regiones, estados, etc.) con alta desigualdad (Hahn et al., 1996). 
Para abordar correctamente tal cuestión, este trabajo sigue una metodología multinivel que permite distinguir los efectos del ingreso individual de los efectos contextuales de desigualdad de ingreso. Las estructuras de datos multinivel son implícitamente jerárquicas al tener anidamientos de unidades "de menor nivel" ( $i)$ dentro de unidades de "mayor nivel" $(j)$, comúnmente denominadas unidades de "nivel-1" y "nivel-2", respectivamente.

Las variables medidas en el nivel-1, siguiendo la notación de Goldstein (1999) y Leeuw \& Meijer (2008), serán denotadas por $x_{i j}$ y las variables medidas en el nivel-2 serán denotadas por $z_{j}$. Dado que el interés es modelar el efecto sobre una variable resultado $y_{i j}$ de las variables de nivel-1 y nivel-2, el modelo multinivel base en este caso es de la forma siguiente:

$\mathrm{y}_{\mathrm{ij}}=\beta_{0 \mathrm{j}}+\beta_{1 \mathrm{j}} \mathrm{x}_{\mathrm{ij}}+\varepsilon_{\mathrm{ij}}$

De forma que $E\left[y_{i j}\right]=\beta_{0 j}+\beta_{1 j} x_{i j}$, bajo el supuesto de que $E\left[\varepsilon_{i j}\right]=0$. Incluyendo la información para las unidades de nivel-2 (parte contextual) y suponiendo que $\beta_{0 j}$ varía aleatoriamente a través de las unidades, el modelo multinivel de intercepto aleatorio con covariables en el nivel-1 y nivel-2 se expresa como:

$\mathrm{y}_{\mathrm{ij}}=\gamma_{00}+\beta_{1 \mathrm{j}} \mathrm{x}_{\mathrm{ij}}+\gamma_{01} \mathrm{z}_{\mathrm{j}}+\delta_{\mathrm{oj}}+\varepsilon_{\mathrm{ij}}$

donde $\gamma_{00}$, muestra el efecto medio a través de la muestra; y $\delta_{0 j}$ representa los residuales del grupo $j$ (nivel-2). Ahora, introduciendo la pendiente aleatoria $\beta_{1 \mathrm{j}}=\gamma_{10}+\delta_{\mathrm{ij}}$, se obtiene el denominado modelo de pendiente aleatoria:

$\mathrm{y}_{\mathrm{ij}}=\gamma_{00}+\gamma_{01} \mathrm{z}_{\mathrm{j}}+\gamma_{10} \mathrm{x}_{\mathrm{ij}}+\delta_{1 \mathrm{j}} \mathrm{x}_{\mathrm{ij}}+\varepsilon_{\mathrm{ij}}$.

Y bajo el supuesto de que la pendiente de regresión está condicionada sobre los factores de nivel-2, el modelo multinivel toma la forma subsiguiente:

$y_{i j}=\left(\gamma_{00}+\gamma_{01} z_{j}+\delta_{0 j}\right)+\left(\gamma_{10}+\gamma_{11} z_{j}+\delta_{1 j}\right) x_{i j}+\varepsilon_{i j}$

$=\gamma_{00}+\gamma_{01} z_{j}+\gamma_{10} x_{i j}+\gamma_{11} z_{j} x_{i j}+\delta_{0 j}+\delta_{1 j} x_{i j}+\varepsilon_{i j}$

En suma, $\gamma_{00}$ corresponde a la estimación del intercepto; $\gamma_{10}$ al coeficiente de la pendiente para la relación entre $x_{i}$ e $y_{i}$ cuando $z_{j}=0 ; \gamma_{01}$ es el coeficiente de pendiente para la relación entre $z_{j}$ e $y_{i}$ cuando $x_{i}=0 ; \gamma_{11}$, corresponde al coeficiente de la interacción entre $x_{i}$ y $z_{j} ; \delta_{l j}$ es el término de perturbación para el coeficiente de pendiente aleatoria $\gamma_{10} ; \delta_{0 j}$ corresponde a la perturbación para el término de intercepto aleatorio, y $\varepsilon_{i j}$ es el término de perturbación de nivel-1. La interacción entre $x_{i}$ y $z_{j}$, al ser medidas en niveles diferentes, se denomina 
interacción a "nivel cruzado". Por lo que el término de perturbación del modelo multinivel es $u_{i j}=\delta_{0 j}+\delta_{1 j} x_{i j}+\varepsilon_{i j}$.

Ahora, generalizando las ecuaciones (1)-(3) para incluir $P$ predictores de nivel-1 y $Q$ predictores de nivel-2, y suponiendo una especificación multinivel en la que $y_{i j}$ es una variable de respuesta binaria (modelo logit multinivel), el modelo será

$\ln \left(\frac{\pi_{i j}}{1-\pi_{i j}}\right)=\gamma_{00}+\sum_{q=1}^{Q} \gamma_{0 q} z_{q j}+\sum_{p=1}^{P} \gamma_{p 0} x_{p i j}+\sum_{q=1}^{Q} \sum_{p=1}^{P} \gamma_{p q} z_{q j} x_{p i j}+\delta_{0 j}+$ $\sum_{p=1}^{P} \delta_{p j} x_{p i j}+\varepsilon_{i j}$

Donde el resultado de interés será la proporción de casos, $\pi_{i j}$, que caen en la categoría 1 del resultado binario medido, y el modelo multinivel para esta proporción está escrito en términos del log-odd ratio (or, o razón de disparidad) (Goldstein, 1999). Es convencional en esta especificación del modelo que $V\left(\varepsilon_{i j}\right)=\sigma^{2}=1$, tal como en los modelos logit tradicionales, y que $E\left[\delta_{0 j}\right]=E\left[\delta_{1 j}\right]=E\left[\varepsilon_{i j}\right]=0 ; V\left[\delta_{0 j}\right]=\tau_{00} ; V\left[\delta_{1 j}\right]=\tau_{11}$.

\section{Datos y población de estudio}

Los datos provienen de la Encuesta Nacional de Calidad de Vida 2008 para Colombia (ECv-2008). Esta encuesta la aplica el Departamento Administrativo Nacional de Estadísticas (DANE) de Colombia. Es representativa para el total nacional y sus nueve regiones. Y tiene una cobertura de 13600 hogares. Adicionalmente, la información sobre las regiones que se incluirá en el segundo nivel del modelo fue obtenida del Ministerio de Protección Social, la Superintendencia Nacional de Salud y otras agencias gubernamentales.

Como variable dependiente se utiliza una medida de salud percibida o $s a$ lud autorreportada. ${ }^{6}$ A partir de la pregunta "Su estado de salud en general es: Muy bueno, Bueno, Regular, o Malo". La medida de salud autorreportada es vista como una apropiada herramienta de vigilancia en salud pública, porque

5 Donde se supone $E\left[\delta_{0 j}\right]=E\left[\delta_{1 j}\right]=E\left[\varepsilon_{i j}\right]=0 ; V\left[\delta_{0 j}\right]=\tau_{00} ; V\left[\delta_{1 j}\right]=\tau_{11} ; V\left[\varepsilon_{i j}\right]=\sigma^{2} ; \operatorname{Cov}\left[\delta_{0 j}, \varepsilon_{i j}\right]=$ $\operatorname{Cov}\left[\delta_{1 j}, \varepsilon_{i j}\right]=\operatorname{Cov}\left[\varepsilon_{i j}, \varepsilon_{k l}\right]=0 ; \mathrm{y} \operatorname{Cov}\left[\delta_{0 j}, \delta_{1 j}\right]=\tau_{01}$ (Goldstein, 1995).

6 Son diferentes las medidas de resultados en salud: van desde medidas subjetivas como salud autopercibida, e indicadores cuasi objetivos de estatus de salud, como la escala SF-36 de funcionamiento físico, indicadores de enfermedad específicos como arteria coronaria, enfermedades limitantes de la movilidad, índice de masa corporal, así como los problemas mentales, hasta medidas como marcadores biológicos. Por su mayor disponibilidad en encuestas socioeconómicas, la medida más utilizada suele ser la salud autorreportada. 
es adecuada para la población, fácil de entender y refleja el estatus de salud de la comunidad, en contraste con el estado de salud de un individuo.

En estudios longitudinales, esta medida subjetiva de salud ha demostrado ser un buen predictor de la mortalidad, en algunos casos, mejor que las medidas físicas. Cuando se trata de estudios para nivel de comunidad, la práctica habitual es reconfigurar la percepción de salud de lo que se denomina salud percibida positiva o autovaloración positiva del estado de salud, que corresponde a las respuestas "muy bueno y bueno" y, lo contrario, autovaloración negativa de la salud o salud percibida negativa o no positiva, que corresponde a las respuestas "regular y malo".

Esta estrategia, aunque no es precisamente incorrecta, debe utilizarse con cuidado, pues el punto de corte que se elige para la dicotomización influiría en los resultados y estadísticamente afectaría la eficiencia. No obstante, en este caso, el punto de corte adoptado para dicotomizar la salud percibida está acorde con las agencias nacionales de investigación y con la mayor parte de la literatura en este tema, lo que permite comparar los resultados con otros estudios. Adicionalmente, cuando se atienden los pesos de las autovaloraciones, se observa que, en cuanto a la valoración positiva, el $63 \%$ percibe su salud como buena (y $9.9 \%$ como muy buena), y en la valoración negativa el $24.1 \%$ la percibe como regular (frente a $2.77 \%$ como mala), por lo que con esta distribución no es aconsejable desde el punto de vista estadístico desagregar los distintos niveles de salud positiva y negativa, ya que los modelos estimados presentarían problemas de fiabilidad y precisión. Así, la variable dicotómica será 0 , para muy bueno y bueno; y 1 para regular y malo; ${ }^{7}$ es decir, se analizará la probabilidad de reportar un estado de salud regular/malo.

\section{Variables independientes}

De nivel individual:

- Sociodemográficas: edad (centrada en la media), género y estatus marital.

- Socioeconómicas: nivel educativo (más alto nivel educativo alcanzado o último grado aprobado); estatus en el mercado de trabajo (¿En qué actividad ocupó la mayor parte del tiempo la semana pasada?: trabajar/no trabajar); y nivel de riqueza individual. Para la medida de riqueza, se construyó un

7 La salud autorreportada es un poderoso predictor, cuya cualidad no varía a través de grupos socioeconómicos (Burstrom \& Fredlund, 2001), además de que es un buen predictor del uso de atención médica (Doorslaer et al., 2000) y de la mortalidad (Doorslaer \& Gerdtham, 2003). 
indicador de estatus socioeconómico (ESE) utilizando componentes principales policóricos, siguiendo a Kolenikov \& Angeles (2009). ${ }^{8}$ Esta es una aproximación para medir la riqueza de las familias por medio de un índice de activos físicos, así el estatus socioeconómico de la familia estará definido en términos de activos físicos o riqueza, y no en términos de ingresos o consumo. Este indicador fue clasificado por quintiles, con el primer quintil para el menor nivel de riqueza y el quintil $\mathrm{V}$ representando el mayor.

- Ambiente socialmente hostil. Se construyeron dos variables a nivel individual: una a partir de la pregunta "Durante los últimos doce meses, de cuál de los siguientes hechos ha sido víctima usted o algún miembro de su hogar: ¿Atraco o robo? Otro hecho violento (homicidio, asesinato, secuestro, lesiones personales, violaciones, extorsiones, desalojos)". Esta pregunta fue codificada como variable dicotómica con valor de 1 si fue víctima y 0 si no. Y una variable de seguridad percibida a partir de la pregunta ¿cómo se siente en el barrio, pueblo o vereda donde vive? Seguro o inseguro. Esta variable se codificó como variable dicotómica, siendo 1 sentirse inseguro y 0 no.

\section{Variables explicativas de nivel-región}

Uno de los principales problemas en la investigación aplicada en Colombia es la dificultad para construir bases de datos confiables con la mayor cobertura posible. Esto obligó a realizar una búsqueda exhaustiva para contar con esa cobertura de los datos, y fue la principal razón para que en este estudio se utilicen, a nivel individual, los datos de la ECV de 2008:

— Desigualdad de ingresos: medido con el coeficiente de Gini regional. Esta medida se utiliza ampliamente, ya que se mueve en un rango de 0 (igualdad absoluta) a 1 (absoluta desigualdad) (MESEP, 2010).

— Ingreso medio de la región: se utiliza el PIB per cápita regional (medido en dólares de 2007) clasificado en terciles como alto, medio y bajo PIB per cápita regional.

- Composición racial: porcentaje de población residente en una región que pertenece a una comunidad indígena.

8 Siguiendo a Kolenikov \& Angeles (2009), se superan las limitaciones del método de Filmer-Pritchett para el manejo de variables de tipo discreto, de igual modo se propone la construcción del indicador de estatus socioeconómico mediante el cálculo de la matriz de correlaciones policóricas, como insumo para el cálculo de los componentes principales. En este caso, como se predice teóricamente, el indicador así construido por lo general se representa con el primer componente principal. 
— Ambiente socialmente hostil: tasa de homicidios en la región por cada cien mil habitantes.

- Espacio público: metros cuadrados de espacio público per cápita, que incluye el total de metros cuadrados disponibles de parques, plazoletas, zonas verdes y áreas de recreo en cada región. Siguiendo a Veenstra (2005) y ante la ausencia de esta medida para Colombia, el espacio público se interpretará como una proxy de capital social contextual.

- Inversión pública: se utiliza el número de médicos por cada diez mil habitantes y el porcentaje de gasto público en educación aplicado en cada región.

\section{Estrategia de estimación}

La hipótesis de trabajo es que la salud se explica mediante variaciones tanto de las características de los individuos como de las regiones. Para tener esto en cuenta, se siguió una estrategia de modelación secuencial en la que la complejidad se incrementó para cada modelo de manera sucesiva.

- Modelo 1 (modelo nulo): consta de dos niveles (individuos en el nivel-1 y región en el nivel-2), con un término constante en la parte fija y aleatoria. Este permite particionar la variación en la salud autopercibida a través de individuos - al interior de las regiones - y entre regiones. Y provee una primera línea base para comparar el tamaño de la variación contextual en la salud autopercibida.

- Modelo 2 (modelo de intercepto aleatorio): igual que el anterior, pero incluye predictores a nivel individual en la parte fija. Se investiga la relación promedio entre reportar salud pobre y los factores sociodemográficos a través de las ocho regiones.

- Modelo 3 (modelo de intercepto aleatorio): igual que el modelo 2, pero incluye otras variables explicativas individuales en la parte fija, relacionadas con el estatus socioeconómico.

- Modelo 3a (modelo de pendiente aleatoria): igual que el modelo 3, pero se explora el efecto de la distribución diferencial del ingreso entre regiones.

- Modelo 4: igual que el modelo 3a y se incluyen tres variables contextuales: una medida de desigualdad de ingreso regional (coeficiente de Gini). Adicionalmente se incluyen interacciones entre niveles de riqueza regional y nivel de desigualdad, buscando identificar los efectos del Gini en las regiones con diferentes niveles de PIB per cápita. Además de que se incluye la variable de composición étnica. 
- Modelo 5: igual que el modelo 4, y se adicionan las variables relacionadas con el ambiente socialmente hostil, las cuales suelen ser consideradas en la interpretación psicosocial.

- Modelo 6: igual que el modelo 4, y se adicionan variables que contrarrestan los ambientes hostiles, como la disponibilidad de espacios para la integración social.

- Modelo 7: igual que el modelo 4, y se adiciona la inversión pública como una variable que puede aproximar la interpretación neomaterialista.

Para ajustar estos modelos se utiliza aproximación de máxima verosimilitud (para facilitar las comparaciones) usando cuadratura adaptativa con siete puntos de integración. Adicionalmente, se utilizó la opción de covarianza no estructurada para permitir que los interceptos aleatorios y las pendientes covaríen (Fielding et al., 2008). Y, finalmente, se calculó para cada modelo el coeficiente de partición de varianza y los estadísticos tradicionales de bondad de ajuste Akaike (AIC), el Criterio de Información Bayesiana (BIC) y la medida de Deviance, los cuales brindan información de mejor ajuste a menor magnitud.

Además, se realizó un test de razón de verosimilitud para verificar la utilización de una pendiente aleatoria. ${ }^{9}$ Los resultados de dicho test indicaron que las pendientes e intercepto en el quintil $V$ de ingreso para salud autopercibida varían entre regiones, por lo que se incluyó una pendiente aleatoria para esta variable.

En resumen, el interés de las estimaciones se centrará en los efectos específicos del coeficiente de Gini sobre la salud percibida. Un modelo que reduzca o haga no significativo el parámetro estimado para el coeficiente de Gini se consideraría que tiene en cuenta alguna o toda la varianza explicada por la desigualdad de ingresos. Siguiendo la notación antes presentada, el modelo por estimar será

$$
\begin{aligned}
& \ln \left(\frac{\pi_{i j}}{1-\pi_{i j}}\right)=\gamma_{00}+\gamma_{01} \text { Gini }_{j}+\gamma_{02}\left(\text { AltoPib }_{j} \times \text { Gini }_{j}\right)+\gamma_{03}\left(\text { MedioPib }_{j} \times \text { Gini }_{j}\right)+ \\
& \gamma_{10} \text { Edad }_{i j}+\gamma_{20} \text { Mujer }_{i j}+\gamma_{30} \text { Trabajar }_{i j}+\gamma_{40} \text { Quintil_II }_{i j}+\gamma_{50} \text { Quintil_III }_{i j}+ \\
& \gamma_{60} \text { Quintil_IV }_{i j}+\gamma_{70} \text { Quintil_V }_{i j}+\delta_{0 j} \text { Quintil_V }_{i j}+\varepsilon_{i j}
\end{aligned}
$$

con $F^{-1}\left(\pi_{i j}\right)$ es el log-odds de que $y_{i j}=1$, es decir, reportar un estado de salud regular/malo.

9 El test de razón de verosimilitud, calculado como dos veces la diferencia entre el valor de log-probabilidad entre el modelo con y sin pendiente aleatoria. El rechazo de la hipótesis nula (que no hay variación entre regiones) implica que hay evidencia de diferencias "reales" entre grupos (regiones), en cuyo caso el modelo multinivel es preferido al de un único nivel. 


\section{Resultados}

Todas las características individuales se especificaron como variables categóricas y la edad fue centrada alrededor de la media de 41 años. El análisis multinivel se realizó sobre 26869 individuos anidados en las ocho regiones en que se divide la geografía colombiana (cuadro 1).

La composición de la población fue de $53 \%$ mujeres y $47 \%$ hombres. El 44\% con educación primaria como máximo logro educativo alcanzado y el $12 \%$ con educación superior. El $66 \%$ de la población consideró que su estado de salud era bueno o muy bueno; mientras que el 34\% lo percibió como regular o malo.

El coeficiente de Gini de 54 ubica al país entre los más desiguales de América Latina, comparado con Perú (49), México (48.3) y Argentina (46); y la alta tasa de homicidio, lo señala entre los más violentos. El PIB per cápita de 2008 en Colombia fue de 4270.54 dólares, muy por debajo de países desarrollados como Dinamarca con 57021 dólares y Estados Unidos con 46341 dólares, y apenas por encima de Perú, con 3807, Bolivia 1386 o Argelia 4005 dólares.

De manera descriptiva, el cuadro 1 muestra la distribución por quintiles de las variables percepción de salud, estatus socioeconómico y educación. En todos los países, con independencia de su nivel de ingresos, la salud y la enfermedad siguen un gradiente social, es decir, cuanto más baja es la situación socioeconómica, peor es el estado de salud. Para Colombia, como se observa en las gráficas subsiguientes, persiste esa relación de gradiente. Según el panel (a), la región de Bogotá presenta los mayores niveles de estatus económico en todos los quintiles de riqueza; esto es, la población del quintil más pobre en Bogotá presenta un estatus económico mayor que la población del mismo quintil en el resto del país. Y lo mismo ocurre con la población del quintil más rico; en esta región residen los más ricos entre los ricos del país. Por su parte, la región Atlántico presenta el menor índice de riqueza para la población del quintil uno, frente a la población del mismo quintil en el resto del país. En esta región residen los más pobres entre los pobres del país.

En el panel (b), por su parte, se verifica que la posibilidad de autopercibir un buen estado de salud se reduce a medida que se desciende en la distribución de la riqueza. O lo que es igual: los quintiles más pobres tienen mayor probabilidad de juzgar su salud como regular o mala, en comparación con la población de los quintiles más ricos.

La región Pacífica se destaca por tener la más baja proporción de la población que autopercibe su salud como buena en todos los quintiles de distribución de la riqueza; mientras que la región Atlántico tiene la más alta proporción. 
Cuadro 1. Estadísticas descriptivas

\begin{tabular}{|c|c|c|}
\hline Variables & Media & $S D$ \\
\hline \multicolumn{3}{|l|}{ Predictores } \\
\hline Mujer & 0.53 & 0.49 \\
\hline Hombre & 0.47 & 0.49 \\
\hline Edad & 41.94 & 17.16 \\
\hline Educación preescolar & 0.09 & 0.29 \\
\hline Educación primaria & 0.44 & 0.49 \\
\hline Educación secundaria & 0.34 & 0.47 \\
\hline Educación superior & 0.12 & 0.32 \\
\hline Casado & 0.61 & 0.48 \\
\hline Soltero & 0.39 & 0.48 \\
\hline Trabaja & 0.96 & 0.19 \\
\hline Quintil I de riqueza & 0.2093 & 0.3995 \\
\hline Quintil II de riqueza & 0.2012 & 0.4009 \\
\hline Quintil III de riqueza & 0.1980 & 0.3985 \\
\hline Quintil IV de riqueza & 0.2047 & 0.4035 \\
\hline Quintil V de riqueza & 0.1969 & 0.3976 \\
\hline Víctima de comportamiento antisocial & 0.01 & 0.11 \\
\hline Percepción de inseguridad & 0.20 & 0.39 \\
\hline Gini & 0.54 & 0.02 \\
\hline Tasa homicidio $\times 100000 \mathrm{~h}$. & 40.38 & 15.88 \\
\hline \% indígena & 4.02 & 4.08 \\
\hline Espacio público (m² per cápita) & 116.77 & 29.68 \\
\hline Gasto educación (\% gasto total) & 7.41 & 3.00 \\
\hline PIB per cápita (dólares 2007) & 4270.52 & 1519.32 \\
\hline Cobertura regional & \multicolumn{2}{|c|}{ \% población } \\
\hline Atlántico & \multicolumn{2}{|l|}{13.42} \\
\hline Oriental & \multicolumn{2}{|l|}{14.03} \\
\hline Central & \multicolumn{2}{|l|}{15.5} \\
\hline Pacífica & \multicolumn{2}{|l|}{16.34} \\
\hline Bogotá & \multicolumn{2}{|l|}{8.18} \\
\hline Amazonía & \multicolumn{2}{|l|}{3.82} \\
\hline Antioquia & \multicolumn{2}{|l|}{13.98} \\
\hline Valle & \multicolumn{2}{|l|}{14.72} \\
\hline
\end{tabular}

En cuanto a juzgar la salud como regular o mala, Bogotá tiene la mayor proporción que autopercibe la salud como mala en el quintil uno, muy alejado de la población del quintil más alto. Este resultado se explicaría en resultados recientes, los cuales indican que en Bogotá el 62\% de la población considera que no se garantiza el derecho a la salud, mientras esta percepción es inferior en un 55\% para el resto de las regiones del país (Bogotá Cómo Vamos, 2013). En Bogotá, más del $54 \%$ de la población en los estratos económicos más bajos 
considera que no hay garantía del derecho a la salud, ello explicaría que, aun cuando en Bogotá se presentan los más altos niveles de riqueza para todos los quintiles, existiría también un limitado acceso a servicios de salud que afectaría la percepción de salud.

Gráfica 1. Gradientes de riqueza y percepción de salud en Colombia

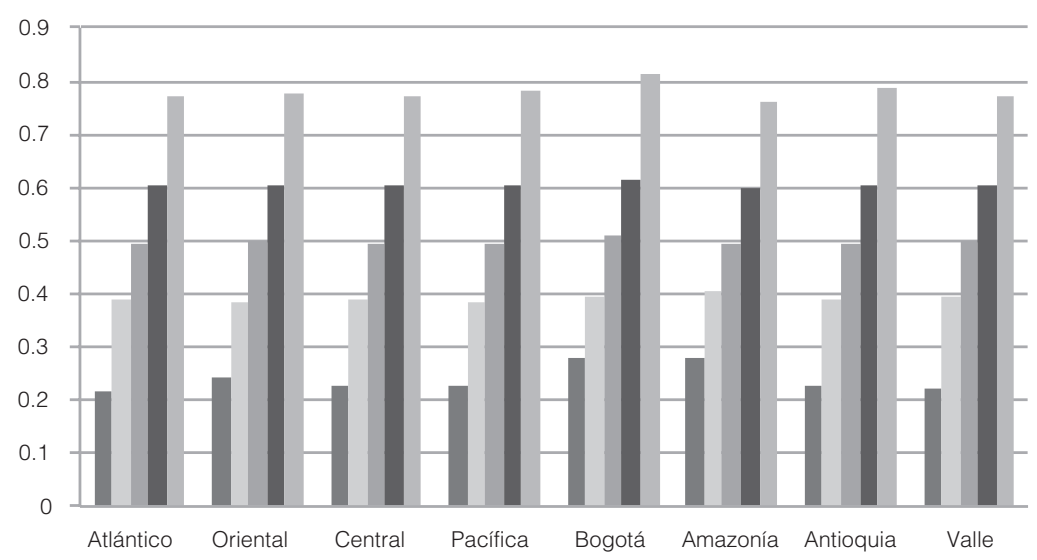

a quintil 1 $\quad$ quintil $2 \quad$ quintil 3 quintil 4 $\quad$ quintil 5

(a) Distribución por quintiles del ESE.

Muy buena/Buena

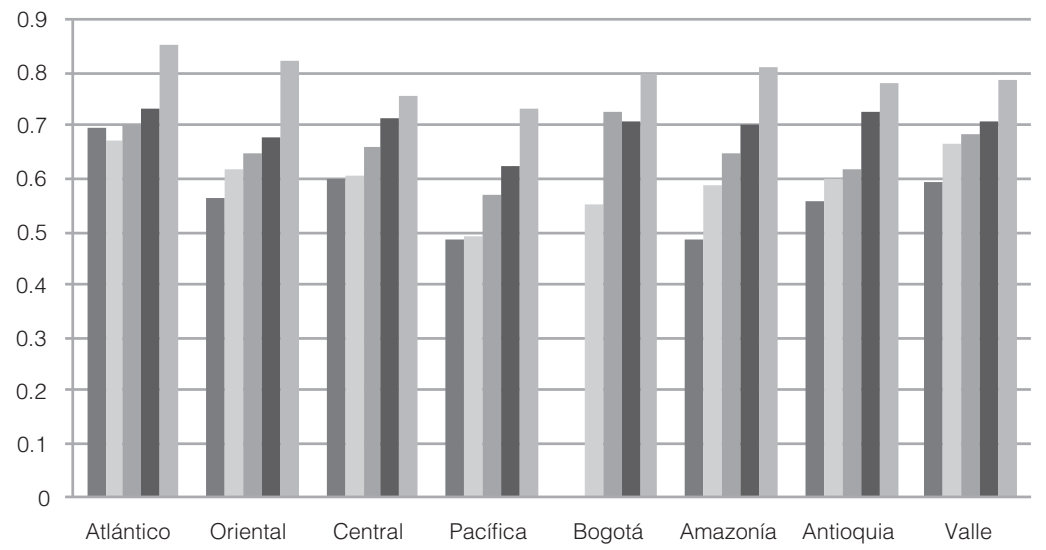

aquintil 1 aquintil 2 quintil 3 quintil 4 quintil 5 


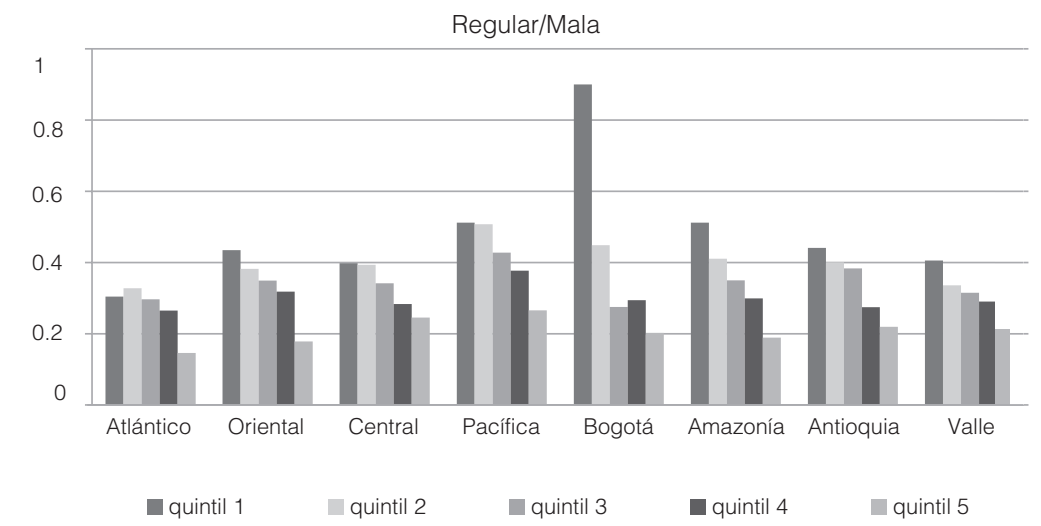

(b) Percepción del estado de salud, según quintiles de riqueza

Fuente: Elaboración propia con base en la ECV-2008 e información obtenida del Ministerio de Protección Social, de la Superintendencia Nacional de Salud y de otras agencias gubernamentales de Colombia.

Salud percibida: ¿se verifica la hipótesis desigualdad de ingreso-salud en Colombia?

Para verificar esta hipótesis, se estimaron varios modelos logit multinivel. El cuadro 2 muestra los resultados de los modelos 1-4. Del modelo 1 se observa, convirtiendo la parte fija de la estimación logit en proporciones, ${ }^{10}$ que el $32 \%$ de la población reporta un pobre estado de salud. Dado que en el modelo 1 no se incluye ningún predictor, se supondría que la probabilidad no varía, en promedio, entre diferentes tipos de personas. Si se toma como grupo de referencia, un hombre de 41 ańos y soltero, la proporción que reporta salud pobre es, en promedio, de $25 \%$. Cuando se consideran las condiciones de estatus socioeconómico, hombre de 41 años, soltero, en el quintil I de la distribución de riqueza y sin trabajo, la proporción se eleva al $38 \%$. Y si se le adiciona el contar con educación universitaria, esta proporción es del 18\%. Los efectos diferenciales independientes (comparados para este grupo de referencia) se muestran abajo, y allí se verá que los efectos para cada una de las variables fueron estimados después de incluir los respectivos controles.

La edad estuvo positivamente asociada con la salud pobre autorreportada, de manera que, a mayor edad, aumenta la probabilidad de reportar un pobre

10 La transformación del logit a proporciones está dada por $e^{\text {Logit }} /\left(1+e^{\text {Logit }}\right)$ o de forma equivalente $1 /\left(1+e^{- \text {Logit }}\right)$ (Goldstein, 1999). 
estado de salud, comparado con la población joven. A partir del modelo 2, también se identifica que es más probable que las mujeres reporten salud pobre en comparación con los hombres, y finalmente no se observan diferencias significativas en el estado marital (estar casado, soltero o divorciado).

Del modelo 2, se observa que la población con menor nivel educativo (preescolar) tienen tres veces más probabilidad $(\mathrm{OR}=3.54)$ de reportar salud pobre (comparados con los de nivel universitario). En general, se verifica una relación de gradiente entre niveles educativos, la probabilidad de reportar un mal estado de salud se reduce a medida que nos movemos a un mayor logro educativo, ensanchando aún más la brecha entre los menos y los más educados.

En el modelo nulo, se identifica una variación significativa en la salud autopercibida a través de las regiones $\left(\operatorname{Var}\left(\delta_{0 j}\right)=0.064\right)$, y esa diferencia se mantiene después de controlar las características socioeconómicas $\left(\operatorname{Var}\left(\delta_{0 j}\right)=0.049\right)$, con lo cual se consideraría que las diferencias regionales en la salud percibida parecen no estar explicadas solo por particularidades individuales; las características del entorno también cuentan. Incluso, en el modelo 3a, al considerar la distribución diferencial de la población del quintil $\mathrm{V}$, las diferencias significativas en la salud autopercibida a través de las regiones se mantiene $\left(\operatorname{Var}\left(\delta_{0 j}\right)=0.051\right)$. En este último caso, es razonable suponer que el mapa de la salud autorreportada para los grupos de alto estatus socioeconómico es diferente de los de bajo estatus.

Mientras que el grupo de bajo estatus socioeconómico es más probable que reporte salud pobre, en promedio, tal vez muestre gran variación, dependiendo de la región de residencia, cuando se les compara con grupos de alto estatus socioeconómico. Así, aun cuando la región es importante para ambos grupos de ingreso, es relativamente más importante para los grupos de bajos ingresos. Pues regiones que sean saludables para grupos de mayor ingreso, pueden no serlo para los más pobres y a la inversa; es el caso de la disponibilidad de infraestructura en salud, la garantía del derecho a la salud y las condiciones medioambientales diferenciales que enfrentaría la población pobre, por ejemplo.

En el modelo 3 se verifica un gradiente significativo de la riqueza para la salud autorreportada. Los individuos del quintil $\mathrm{V}$ tienen la menor probabilidad diferencial $(\mathrm{OR}=0.45)$ de reportar su estado de salud como pobre frente a una persona del quintil I; seguidos por la población ubicada en el IV $(\mathrm{OR}=0.69)$, la del III $(\mathrm{OR}=0.83)$ y el II $(\mathrm{OR}=0.96)$. Lo cual insiste en la importancia de la hipótesis de ingreso absoluto y de otros factores socioeconómicos individuales para explicar la salud de las personas.

Hasta este punto aún permanece la varianza en la salud percibida, que no se explica solo por las características del individuo, por ello, luego de introducir la pendiente para el quintil $V$ de riqueza, en el modelo 4 se exploran los factores contextuales que se espera expliquen el resto de esta varianza en la autopercepción de 
salud. Específicamente se evalúa el impacto de variables contextuales, como la composición étnica, la riqueza media de la región y los niveles de desigualdad.

En cuanto al ingreso per cápita, esta es una importante variable, ya que suele capturar la riqueza general de las regiones y por esta vía la disponibilidad de recursos. Algunos autores han encontrado que las medidas de bienestar (salud física, mental, entre otras), están fuertemente influenciadas por el pIB per cápita y que suele existir una fuerte y pronunciada interacción entre el pIB y el coeficiente de Gini. De acuerdo con esto, en el modelo 4 se ajusta la riqueza de la región con la inclusión de una interacción entre el PIB per cápita (por quintiles) y el coeficiente de Gini regional. La interacción entre este y el pIB significa que ahora el coeficiente de Gini hará referencia principalmente al efecto que la desigualdad tiene dentro de las regiones del primer quintil de riqueza (categoría de referencia).

Cuadro 2. Estimación parte fija y aleatoria para el modelo analítico multinivel de la percepción de estado de salud (en logits)

\begin{tabular}{|c|c|c|c|c|c|}
\hline \multirow[t]{2}{*}{ Variables } & \multicolumn{5}{|c|}{ Modelos } \\
\hline & 1 & 2 & 3 & $3^{a}$ & 4 \\
\hline \multirow[t]{2}{*}{ Edad } & & $0.0362^{* * *}$ & $0.0401^{* * *}$ & $0.0401^{* * *}$ & $0.0401^{* * *}$ \\
\hline & & $(0.000899)$ & $(0.000947)$ & $(0.000947)$ & $(0.000947)$ \\
\hline \multirow[t]{2}{*}{ Mujer } & & $0.454^{\text {***}}$ & $0.486^{\star \star \star}$ & $0.486^{\star \star \star}$ & $0.486^{* * *}$ \\
\hline & & $(0.0287)$ & $(0.0291)$ & $(0.0291)$ & $(0.0291)$ \\
\hline \multirow[t]{2}{*}{ Casado } & & 0.0444 & 0.0528 & 0.0532 & 0.0523 \\
\hline & & $(0.0297)$ & $(0.0300)$ & $(0.0300)$ & $(0.0300)$ \\
\hline \multirow[t]{2}{*}{ Preescolar } & & $1.731^{\star \star \star *}$ & $1.265^{\star \star \star}$ & $1.263^{\text {***}}$ & $1.266^{\star \star *}$ \\
\hline & & $(0.0705)$ & $(0.0768)$ & $(0.0769)$ & $(0.0769)$ \\
\hline \multirow[t]{2}{*}{ Primaria } & & $1.481^{\text {***}}$ & $1.099^{* * *}$ & $1.097^{\star \star *}$ & $1.102^{\star \star \star}$ \\
\hline & & $(0.0585)$ & $(0.0635)$ & $(0.0636)$ & (0.0636) \\
\hline \multirow[t]{2}{*}{ Secundaria } & & $0.864^{\star * \star}$ & $0.647^{\star \star \star}$ & $0.645^{\star \star \star}$ & $0.649^{* * *}$ \\
\hline & & $(0.0606)$ & $(0.0625)$ & $(0.0626)$ & $(0.0626)$ \\
\hline \multirow[t]{2}{*}{ Quintil II } & & & -0.0373 & -0.0366 & -0.0353 \\
\hline & & & $(0.0433)$ & $(0.0434)$ & $(0.0433)$ \\
\hline \multirow[t]{2}{*}{ Quintil III } & & & $-0.178^{\star \star \star}$ & $-0.177^{\star \star \star}$ & $-0.175^{\star \star \star}$ \\
\hline & & & $(0.0455)$ & $(0.0456)$ & $(0.0456)$ \\
\hline \multirow[t]{2}{*}{ Quintil IV } & & & $-0.362^{\star \star *}$ & $-0.360^{\star \star \star}$ & $-0.361^{\star \star \star}$ \\
\hline & & & $(0.0484)$ & $(0.0486)$ & $(0.0484)$ \\
\hline \multirow[t]{2}{*}{ Quintil V } & & & $-0.784^{\star \star \star}$ & $-0.786^{\star \star \star}$ & $-0.787^{\star \star \star}$ \\
\hline & & & $(0.0566)$ & $(0.0598)$ & $(0.0601)$ \\
\hline \multirow[t]{2}{*}{ Trabaja } & & & $-0.165^{\star}$ & $-0.165^{\star}$ & $-0.164^{\star \star}$ \\
\hline & & & $(0.0764)$ & $(0.0764)$ & $(0.0764)$ \\
\hline \multirow[t]{2}{*}{ Indígena } & & & & & 0.0162 \\
\hline & & & & & $(0.0333)$ \\
\hline \multirow[t]{2}{*}{ Alto PIB $\times$ Gini } & & & & & -0.712 \\
\hline & & & & & $(0.510)$ \\
\hline
\end{tabular}


Cuadro 2. Estimación parte fija y aleatoria para el modelo analítico multinivel de la percepción de estado de salud (en logits) (Continuación)

\begin{tabular}{|c|c|c|c|c|c|}
\hline \multirow[t]{2}{*}{ Variables } & \multicolumn{5}{|c|}{ Modelos } \\
\hline & 1 & 2 & 3 & $3^{a}$ & 4 \\
\hline \multirow[t]{2}{*}{ Medio PIB $\times$ Gini } & & & & & -0.00647 \\
\hline & & & & & $(0.454)$ \\
\hline \multirow[t]{2}{*}{ Gini } & & & & & $6.907^{\star \star}$ \\
\hline & & & & & (3.167) \\
\hline \multirow[t]{2}{*}{ Constante } & $-0.73^{\star \star \star}$ & $-2.209^{\star \star *}$ & $-1.508^{\star * *}$ & $-1.507^{\star \star \star}$ & $-5.408^{* * *}$ \\
\hline & $(0.091)$ & $(0.107)$ & $(0.129)$ & $(0.130)$ & $(1.648)$ \\
\hline \multicolumn{6}{|c|}{ Parámetros de efectos aleatorios } \\
\hline \multicolumn{6}{|c|}{ Varianza de nivel-2 } \\
\hline \multirow[t]{2}{*}{$\operatorname{var}(\delta 0 j)$} & 0.0648035 & 0.0622363 & 0.0497044 & 0.0510213 & 0.0277119 \\
\hline & $(0.0331124)$ & $(0.0318374)$ & $(0.0255614)$ & $(0.0263054)$ & $(0.0160496)$ \\
\hline \multicolumn{6}{|c|}{ Parámetros aleatorios } \\
\hline \multirow[t]{2}{*}{ var(Quintil_V) } & & & & 0.0022636 & 0.0025451 \\
\hline & & & & $(0.0076355)$ & $(0.0057473)$ \\
\hline \multirow[t]{2}{*}{$\operatorname{cov}\left(\right.$ Quintil_V, $\left.\delta_{0 j}\right)$} & & & & -0.006395 & 0.0083982 \\
\hline & & & & $(0.011305)$ & $(0.0105752)$ \\
\hline \multicolumn{6}{|c|}{ Medidas de variación } \\
\hline AIC & 33946.76 & 29717.33 & 29481.19 & 29487.36 & 29491.03 \\
\hline $\mathrm{BIC}$ & 33963.15 & 29782.92 & 29587.78 & 29610.34 & 29646.8 \\
\hline Deviance & 33942.756 & 29701.327 & 29455.192 & 29457.357 & 29453.025 \\
\hline Observaciones & 26869 & 26869 & 26869 & 26869 & 26869 \\
\hline
\end{tabular}

Nota: $n=26869$ individuos anidados en ocho regiones. Por ausencia de información contextual, no se incluyó en este análisis la región San Andrés y Providencia. Para las variables categóricas, el grupo de referencia es 41 años, hombre, quintil I, desempleado y bajo PIB per cápita. ${ }^{*} P<0.05,{ }^{\star \star} P<0.01,{ }^{\star \star \star} P<0.001$. Errores estándar entre paréntesis.

Fuente: Elaboración propia utilizando la ECV-2008.

Se observa una relación negativa entre la probabilidad de juzgar el estado de salud como malo, cuando se vive en una zona de alto y medio PIB, respecto de vivir en una zona de bajo PIB, cuando la desigualdad aumenta. Lo cual estaría en la línea de algunas investigaciones que encuentran que los grupos de altos ingresos tienden a estar mejor en sociedades más desiguales (Subramanian et al., 2001). Sin embargo, estas variables parecen no ser por sí solas significativas para explicar el estado de salud de la población, y aún persisten diferencias significativas en la salud autopercibida entre regiones $\left(\operatorname{Var}\left(\delta_{0 j}\right)=0.028\right)$.

El coeficiente de Gini es positivo y significativo, indicando que la mayor desigualdad se relaciona positivamente con la probabilidad de autopercibir el estado de salud como regular o malo. Lo cual apoyaría la hipótesis de desigualdad de ingreso. Se observa que aun controlando el logro educativo, el coeficiente de Gini sigue siendo significativo y muestra una relación positiva con la probabilidad de reportar un pobre estado de salud. Con lo que el logro educativo no sería un factor de confusión de la relación entre desigualdad y salud pobre; 
asimismo, se mantiene el significativo gradiente en la relación entre logro educativo y pobre salud autopercibida.

El modelo 4 explora el efecto de la composición racial; si bien este presenta un signo positivo, indicando que en regiones con mayor proporción de población indígena aumenta la probabilidad de juzgar la salud como regular o mala (en línea con Subramanian \& Kawachi, 2004; Subramanian et al., 2002, para Estados Unidos), no fue significativo para explicar la percepción de salud. La consideración de esta variable no afectó la significancia del parámetro de desigualdad, por lo cual no actúa como un factor que confunda la relación desigualdad de ingreso-salud. Incluso al agregar tanto la educación como la pertenencia étnica, el coeficiente de Gini sigue siendo significativo para explicar la percepción de regular/mala salud de la población.

Debido a que el modelo 4 ya se controla por los principales factores considerados en la literatura como distorsionadores de la relación salud-desigualdad (ingreso individual, logro educativo y composición racial), el hecho de que la riqueza para los diferentes quintiles siga siendo significativa y no presente mayores variaciones en cuanto a la magnitud de sus parámetros antes y después de la inclusión de la variable de desigualdad, permite intuir que el efecto de esta sobre la salud va más allá del estatus socioeconómico.

¿Qué factores median la asociación entre desigualdad y pobre autopercepción de salud?

A partir de las asociaciones identificadas en los modelos 5 a 7 (cuadro 3), se exploran las hipótesis que buscan explicar el vínculo entre desigualdad de ingreso y salud pobre. En el cuadro 3 se toma el modelo 4 como referencia, dado que no hay cambios significativos en los parámetros de características individuales, se muestran solo los resultados del haber considerado las nuevas variables de análisis. En el modelo 5 se introduce la percepción de inseguridad y el haber sido víctima de comportamiento antisocial, además se encuentra que estas variables que aproximan el ambiente socialmente hostil son significativas para explicar una pobre percepción de salud. Así, que un individuo perciba su entorno como inseguro, aumenta la probabilidad de juzgar su estado de salud como malo $(\mathrm{OR}=1.6)$; pero, contrario a lo que se esperaría, el haber sido víctima de comportamiento antisocial presenta una relación negativa con juzgar el estado de salud como regular o malo, una intuición para este resultado sería la disponibilidad de acceso a servicios cuando se es víctima de hechos violentos, dado que la atención de urgencias es obligatoria y, por ende, el rápido acceso (o acceso real) distorsionaría la percepción de salud (acceso potencial). La 
inclusión de estas variables redujo el coeficiente de Gini y su significancia. Por lo cual se podría considerar que las variables relacionadas con la hostilidad del ambiente dan cuenta parcialmente del efecto del coeficiente de Gini sobre la percepción del estado de salud, aunque este sea significativo.

El modelo 6 muestra que la mayor disponibilidad de espacios públicos reduce la probabilidad de juzgar el estado de salud como regular/malo $(\mathrm{OR}=0.99)$, lo que estaría en línea con la perspectiva psicosocial, según la cual la disponibilidad de espacios para la integración social contrarrestaría los efectos de la desigualdad, facilitaría la generación de capital social y mejoraría la percepción de salud. Además, la inclusión de esta variable reduce el parámetro para el coeficiente de Gini y además lo vuelve no significativo; pero haciéndose significativo el efecto de la desigualdad en las regiones de PIв medio.

Lo anterior sugiere que la variable de espacio público para la interacción social da cuenta del efecto de la desigualdad de ingreso sobre la salud autopercibida en las regiones de riqueza media. Conforme la desigualdad aumenta, menor es la probabilidad de juzgar la salud como regular o mala cuando se reside en una región de riqueza media, respecto de alguien que vive en una región de baja riqueza. Este resultado, incluso con las limitaciones de la medida disponible, estaría en línea con la visión epidemiológica, la cual ve en el contexto en que reside el individuo una explicación a su estado de salud.

Por último, en el modelo 7 se incluye el porcentaje de gasto en educación, buscando explorar la denominada perspectiva neomaterialista. ${ }^{11}$ En este caso, la teoría prevé que el mayor gasto público (en educación) reduce la probabilidad de percibir el estado de salud regular/malo, lo que se verifica en estos resultados $(\mathrm{OR}=0.94)$. Se aminora el impacto de la desigualdad, y el impacto específico de esta sobre las regiones con PIB per cápita medio sigue siendo significativo. Lo cual refleja que esta variable también controla los efectos de la desigualdad. Aunque siguen siendo significativos para explicar la percepción de salud de la población.

Los resultados de los modelos 5 a 7 sugieren que las variables que aproximan la perspectiva psicosocial serían las más satisfactorias para explicar el efecto de la desigualdad de ingreso en los datos, frente a la perspectiva neomaterialista, en términos de alterar los coeficientes relacionados con el Gini. Y a partir de los estadísticos AIC у в вIC se observa que el modelo 5 presenta el mejor ajuste para los datos frente al modelo 6 . Lo cual concordaría con la hipótesis de desigualdad de ingreso y salud, en cuanto a evidenciar que las áreas sometidas a mayor desigualdad afectan la salud de los individuos vía ambientes socialmente hostiles

11 Desde esta perspectiva, se realizaron ejercicios incluyendo número de médicos por cada diez mil habitantes y el gasto en salud, pero se presentaron problemas de multicolinealidad y se eliminaron del análisis. 
(Widman, 2001, 2003). Pero también indica que la riqueza individual sigue siendo importante para garantizar el acceso, y que el impacto sobre este último es diferencial, dependiendo de si el individuo vive en zonas de mayor PIB per cápita o no. Con lo cual, si bien las variables que aproximan el ambiente social hostil, o perspectiva psicosocial, son significativas para influir la percepción de salud, la riqueza individual y el entorno en que vive el individuo sigue pesando al momento de garantizar una mejor salud.

Cuadro 3. Estimación parte fija y aleatoria del modelo analítico multinivel de la percepción de estado de salud, considerando hipótesis que vinculan la desigualdad de ingresosalud (en logits)

\begin{tabular}{|c|c|c|c|c|}
\hline \multirow[t]{2}{*}{ Variables } & \multicolumn{4}{|c|}{ Modelos } \\
\hline & 4 & 5 & 6 & 7 \\
\hline \multirow[t]{2}{*}{ Alto PIB $\times$ Gini } & -0.712 & -0.283 & -0.723 & -0.0988 \\
\hline & $(0.510)$ & $(0.509)$ & $(0.401)$ & $(0.228)$ \\
\hline \multirow[t]{2}{*}{ Medio PIB x Gini } & -0.00647 & -0.572 & $-1.109^{* * *}$ & $-0.648^{* *}$ \\
\hline & $(0.454)$ & $(0.420)$ & $(0.376)$ & $(0.209)$ \\
\hline \multirow[t]{2}{*}{ Gini } & $6.907^{\star \star}$ & $6.237^{\star}$ & 0.854 & $5.431^{\star \star \star}$ \\
\hline & $(3.167)$ & $(3.091)$ & (2.994) & $(1.609)$ \\
\hline \multirow[t]{2}{*}{ Víctima comportamiento antisocial } & & $-0.262^{*}$ & & \\
\hline & & $(0.131)$ & & \\
\hline \multirow[t]{2}{*}{ Percepción inseguridad } & & $0.464^{\star \star \star}$ & & \\
\hline & & $(0.0360)$ & & \\
\hline \multirow[t]{2}{*}{ Espacio público } & & & $-0.00778^{\star \star}$ & \\
\hline & & & $(0.00301)$ & \\
\hline \multirow[t]{2}{*}{ Gasto educación } & & & & $-0.0545^{\star \star \star}$ \\
\hline & & & & $(0.0119)$ \\
\hline \multirow[t]{2}{*}{ Constante } & $-5.408^{* * *}$ & $-4.727^{\star *}$ & -0.583 & $-3.917^{* * *}$ \\
\hline & $(1.648)$ & $(1.608)$ & $(1.928)$ & $(0.857)$ \\
\hline \multicolumn{5}{|l|}{ Parámetros de efectos aleatorios } \\
\hline \multicolumn{5}{|l|}{ Varianza de nivel-2 } \\
\hline $\operatorname{var}(\delta 0 j)$ & $\begin{array}{c}0.0277119 \\
(0.0160496)\end{array}$ & $\begin{array}{c}0.0363972 \\
(0.0228973)\end{array}$ & $\begin{array}{c}0.0305129 \\
(0.0192425)\end{array}$ & $\begin{array}{c}0.012612 \\
(0.0085454)\end{array}$ \\
\hline \multicolumn{5}{|l|}{ Parámetros aleatorios } \\
\hline var(Quintil_V) & $\begin{array}{c}0.0025451 \\
(0.0057473)\end{array}$ & $\begin{array}{c}0.0039951 \\
(0.0078673)\end{array}$ & $\begin{array}{c}0.0116143 \\
(0.0119462)\end{array}$ & $\begin{array}{c}0.0090656 \\
(0.0098714)\end{array}$ \\
\hline $\operatorname{cov}\left(\right.$ Quintil_V, $\left.\delta_{o j}\right)$ & $\begin{array}{c}0.0083982 \\
(0.0105752)\end{array}$ & $\begin{array}{c}-0.012059 \\
(0.0144491)\end{array}$ & $\begin{array}{c}-0.018825 \\
(0.0141771)\end{array}$ & $\begin{array}{c}-0.010693 \\
(0.0086193)\end{array}$ \\
\hline \multicolumn{5}{|l|}{ Medidas de variación } \\
\hline AIC & 29491.03 & 29329.69 & 29487.88 & 29480.76 \\
\hline $\mathrm{BIC}$ & 29646.8 & 29501.86 & 29651.85 & 29644.74 \\
\hline Deviance & 29453.025 & 29287.692 & 29447.879 & 29440.764 \\
\hline Observaciones & 26869 & 26869 & 26869 & 26869 \\
\hline
\end{tabular}

Nota: Individuos anidados en ocho regiones. No se incluye las regiones de San Andrés y Providencia. Para las variables categóricas, el grupo de referencia es 41 años, hombre, educación superior, quintil I, desempleado, bajo PIB per cápita, no pertenecer a una comunidad indígena, haber sido víctima de comportamiento antisocial y sentirse inseguro en el lugar de residencia. ${ }^{*} \mathrm{P}<0.05,{ }^{* \star} \mathrm{P}<0.01,{ }^{\star \star *} \mathrm{P}<0.001$. Errores estándar entre paréntesis.

Fuente: Elaboración propia a partir de la ECV-2008. 
Adicionalmente, la inclusión de las variables de los modelos 5-7 muestran que la variable de segundo nivel deja de ser significativa, lo cual es una evidencia a favor de la importancia de las variables consideradas en el nivel contextual para explicar cómo las variables de entorno explicarían el fenómeno de autopercepción de salud.

\section{Discusión y conclusiones}

Este trabajo se planteó dos propósitos: 1) verificar que las condiciones económicas contextuales ejercen influencia sobre la salud autopercibida, independientemente del estatus socioeconómico en el que se ubique el individuo. Por lo que se buscaba diferenciar los efectos del ingreso del individuo de los efectos contextuales de la desigualdad de ingresos, sobre la salud autopercibida. Se mostró que, si bien el estatus socioeconómico es un determinante esencial de la salud de la población a través de las regiones, permanecen diferencias significativas, incluso después de controlar la riqueza individual; y también permanecen las diferencias luego de controlar el nivel educativo y pertenencia étnica. Asimismo, después de controlar el rubro de la riqueza de las regiones medida por el pIB per cápita, se observa que permanecen variaciones significativas en la salud a nivel contextual. Estas solo desaparecen cuando se considera la medida de desigualdad de ingreso. Y 2) se buscó explorar los mecanismos que operarían en el nivel de las regiones y que coadyuvarían a explicar la influencia de las condiciones económicas estructurales sobre la percepción de una salud pobre. Además de intentar aportar a la literatura que ha centrado la atención en el poco cuidado puesto en los trabajos empíricos que investigan las posibles explicaciones a los efectos contextuales (Macintyre \& Ellaway, 2000).

Se ha presentado un modelo exploratorio de los vínculos entre el estatus socioeconómico y la desigualdad de ingresos de las regiones con la salud autopercibida, contrastado de manera sistemática. Las hipótesis abordadas, relacionadas con ingreso individual, ambiente psicosocial y la interpretación neomaterialista, evidencian que el vínculo entre pobre salud autopercibida, con la desigualdad de ingreso, se daría, vía el ambiente psicosocial, más que por medio del ingreso individual o desde la perspectiva neomaterialista.

Las variables para contrastar la hipótesis de ambiente psicosocial reducen el coeficiente de Gini en magnitud y significancia, a la vez que mantienen no significativos los efectos cruzados entre este y el pIB per cápita. Aun cuando el modelo con las variables que representan un ambiente socialmente hostil presenta un mejor grado de ajuste, resulta interesante observar que el modelo que incluye el espacio público (que se pensaría como una proxy de capital social 
contextual) aumenta la significancia del efecto de la desigualdad sobre las regiones de PIB per cápita medio. Esto sugeriría que los mecanismos a través de los cuales la desigualdad de ingresos influye en la percepción del estado de salud de la población, varía dependiendo de la riqueza de las regiones.

Entre las principales limitaciones de este estudio se señala, en primer lugar, que si bien este trabajo se encuentra en la denominada tercera fase de la investigación entre desigualdad de ingreso-salud por Wagstaff y Doorslaer, dado que utiliza información de individuos, la agregación de la encuesta no permite, en la parte contextual, definir áreas geográficas menores a la regional. Con lo que se hubiese podido verificar si el vínculo desigualdad de ingreso-salud permanece a nivel de ciudades, por citar un ejemplo. En segundo lugar, cabe subrayar que los individuos no se localizan aleatoriamente en las diferentes regiones. Hay un inevitable elemento de autoselección en términos de dónde seleccionan vivir ellos; no obstante, es improbable corregir completa y satisfactoriamente este tipo de situaciones.

Esta investigación se ha centrado en la utilización de la modelación multinivel para describir la existencia de factores contextuales que expliquen la percepción de un pobre estado de salud. Así, el proceso de inclusión de variables de control buscó afinar la descripción de la relación entre las características sociodemográficas y el estatus socioeconómico individual, con variables a nivel de contexto, minimizando los problemas de sesgo de variable omitida y de endogeneidad. Así pues, la investigación empírica sobre si las relaciones encontradas son causales o no, no forman parte del alcance de este trabajo. No obstante, una característica relevante de la modelación multinivel es que al incluir los dos tipos de residuos en la estimación de efectos aleatorios se controla, en parte, el problema de endogeneidad mediante el control estadístico de los efectos de los factores no observables, los cuales influyen en los individuos y las regiones, así como en los resultados.

Adicionalmente, se realizó el test de Hausman para verificar la ausencia de endogeneidad de nivel II, y se rechazó la hipótesis de que algunas características no observables de las regiones que se relegan al término de error estuvieran correlacionadas con características observables del individuo (Grilli \& Rampichini, 2006). ${ }^{12}$ Aun considerando estas limitantes, los resultados del presente trabajo están en la línea de la literatura reciente que intenta aproximarse a los

12 El test de Hausman se desarrolló con el fin de comparar el modelo de efectos fijos — que contenía solo características individuales - con el modelo de efectos aleatorios que contenía características individuales y regionales. La hipótesis nula es que los efectos aleatorios no están correlacionados con ninguna de las variables observables del individuo. El resultado del test de Hausman para detectar endogeneidad de nivel II Prob>Chi2=0.9 rechaza los efectos fijos a favor de los efectos aleatorios y, por ende, se consideraría que los coeficientes estimados son consistentes. 
mecanismos que subyacen en la relación desigualdad de ingreso-salud, sobre todo constituye el primer esfuerzo en este sentido para el caso de Colombia, que intenta aproximar esta relación. Como se mostró en la revisión de la literatura, el amplio conocimiento sobre esta relación ha sido documentado para países o regiones desarrollados (Estados Unidos, Inglaterra y la Unión Europea), mientras que en los países en vías de desarrollo, como Colombia, se ha dado por hecho que deberían operar en un mayor nivel los factores relacionados solo con el ingreso individual.

Finalmente, en términos de la política pública, se observa que, como pasa con las recomendaciones en las economías en vías de desarrollo, el único mecanismo para incidir en la salud de la población no es el gasto público, o las transferencias directas de ingreso. Subyacente en las características de las regiones, se encuentra una condición de desigualdad que de igual modo impacta en las condiciones de acceso y autopercepción de la salud, que deberá igualmente ser atacada con la intervención del Estado. Como lo señala el informe del PNUD (2010) sobre desarrollo humano para América Latina, es posible romper la tendencia de la desigualdad, pero para lograrlo es necesario actuar en el ámbito de los hogares y en el contexto inmediato en el que estos se desenvuelven, así como en el sistema de redistribución y regulación por parte del Estado.

\section{Referencias}

Anand, S. \& Ravallion, M. (1993). Human Development in Poor Countries: On the Role of Private Incomes and Public Services. J. Econ. Perspect., 7(1), 133-150.

Bernal, R. \& Cardenas, M. (2005, enero). Race and Ethic Inequality in Health and Health Care in Colombia. Fedesarrollo. Working papers, (29).

Bidani, B. \& Ravallion, M. (1997). Decomposing Social Indicators Using Distributional Data. J. Econometrics, 77(1), 125-140.

Bogotá Cómo Vamos. (2013). Encuesta de percepción ciudadana, Bogotá 2013. Bogotá: Bogotá Cómo Vamos.

Bowles, S. \& Gintis, H. (2002). The Inheritance of Inequality. Journal of Economic Perspectives, 16(3), 3-30.

Burstrom, B. \& Fredlund, P. (2001). Self-Rated Health: Is it as Good a Predictor of Subsequent Mortality among Adults in Lower as well as in Higher Social Classes? Journal of Epidemiology and Community Health, (55), 836-840. 
Carrin, G. \& Polti, C. (1995). Exploring the Health Impact of economic Growth, Poverty Reduction and Public Health Expenditure. Tijdschr. Econ. Manage, 15(3-4), 227-246.

Case, A., Fertig, A. \& Paxton, C. (2005). The Lasting Impact of Childhood Health and Circumstance. Journal of Health Economics, (24), 365-389.

Castaño, R. et al. (2000). Evolución de la equidad en el sistema colombiano de salud. Final Report to ECLAC.

Cepal. (2009, 2013). Panorama social de América Latina. Santiago de Chile: onu.

Céspedes, J., Jaramillo, I., \& Castańo, R. (2001). Impacto de la reforma del sistema de seguridad social sobre la equidad en los servicios de salud en Colombia. Artículo preliminar.

CIE, Universidad de Antioquia (2001, diciembre). Observatorio de la seguridad social. Medellín: Grupo de Economía de la Salud (GEs), 1(3).

Cohen, S., Line, S., Manuck, S., Rabin, B., Heise, E. \& Kaplan, J. (1997). Chronic Social Stress, Social Status, and Susceptibility to Upper Respiratory Infections in Nonhuman Primates. Psychosomatic Medicine, 59(3), 213-221.

Cohen, S., Tyrrell, D. \& Smith, A. (1991). Psychological Stress and Susceptibility to the Common Cold. New England Journal of Medicine, 325(9), 606-612.

Contoyannis, P., Jones, A. \& Rice, N. (2004). The Dynamics of Health in the British Household Panel Survey. Journal of Applied Econometrics, (19), 453-503.

Contoyannis, P. \& Forster, M. (1999). The Distribution of Health and Income: A Theoretical Framework. Journal of Health Economics, (18), 605-622.

Costa-Font, J. \& Hernández-Quevedo, C. (2012). Measuring Inequalities in Health: What do We Know? What do We Need to Know? Health Policy, (106), 195-206.

Daly, M., Duncan, G., Kaplan, G. \& Lynch, J. (1998). Macro-to-Micro Links in the Relationship Between Income Inequality and Mortality. Mill Bank Quarterly, 76(3), 315-339.

Davey-Smith, G. (1996). Income Inequality and Mortality: Why are they Related? British Medical Journal, (313), 987-988.

Deaton, A. (2003). Health, Inequality, and Economic Development. Journal of Economic Literature, (41), 113-158. 
Doorslaer, E. van et al. (2000). Equity in the Delivery of Health Care in Europe and the US. Journal of Health Economics, (19), 553-583.

Doorslaer, E. van et al., (1997). Income-Related Inequalities in Health: Some International Comparisons. Journal of Health Economics, (16), 93-112.

Doorslaer, E. van \& Gerdtham, U-G. (2003). Does Inequality in Self-Assessed Health Predict Inequality in Survival by Income? Evidence from Swedish Data. Social Science and Medicine, (57), 1621-1629.

Fielding, A., Pillinger, R., Steele, F. \& Rasbash, J. (2008). Learning Environment for Multilevel Methodology and Applications. Bristol: University of Bristol: Centre for Multilevel Modelling.

Fiscella, K. \& Franks, P. (2000). Quality, Outcomes, and Satisfaction: Individual Income, Income Inequality, Health, and Mortality: What are and the Relationships? Health Services Research, 35(1), 307-318.

Fiscella, K. \& Franks, P. (1997). Poverty or Income Inequality as Predictor of Mortality: Longitudinal Cohort Study. BMJ, (314), 17241727.

Flegg, A. (1982). Inequality of Income, Illiteracy, and Medical Care as Determinants of Infant Mortality in Developing Countries. Population Studies, 36(3), 441-458.

Florez, C. \& Tono, T. (2002). Inequities in Health Status and Use of Health Services in Colombia 1990-2000. Cicred's Seminar.

Flórez, C. \& Nupia, O. (2001). Tendencias en las inequidades en el acceso a los servicios de salud en la población colombiana. 1990-2000. Bogotá: Final report to Fundación Corona/CEDE/ Centro de Gestión Hospitalaria (Monografía, 1).

Gerdtham, U-G. \& Johannesson, M. (2004). Absolute Income, Relative Income, Income Inequality and Mortality. Journal of Human Resources, 39(1), 228-244.

Goldstein, H. (1995, 1999). Multilevel Statistical Models. Londres: Edward Arnold.

Gravelle, H. (1998). How much of the Relation between Population Mortality and Unequal Distribution of income is a Statistical Artefact? British Medical Journal, (314), 382-385.

Grilli, L. \& Rampichini, C. (2006). Model Building Issues in Multilevel Linear Models with Endogenous Covariates. Florencia: Dipartimento di Statistica, Università di Firenze Working Paper. 
Grossman, M. (2000). The Human Capital Model. En Culyer, A. J. \& Newhouse, J. P. (Eds.). Handbook of Health Economics (pp. 347-408). Ámsterdam: Elsevier.

Hahn, R., Eaker, E., Barker, N., Teutsch, S., Sosniak, W. \& Krieger, M. (1996). Poverty and Death in the United States. International Journal of Health Services, (26), 673-690.

Humphries, K. \& Doorslaer, E. van. (2000). Income-Related Health Inequality in Canada. Social Science and Medicine, (50), 663-671.

Jones, A. \& Wildman, J. (2008). Health, Income and Relative Deprivation: Evidence from the BHP. Journal of Health Economics, 27(2), 308-324.

Kakwani, N., Wagstaff, A. \& Doorslaer, E. van. (1997). Socioeconomic Inequalities in Health: Measurement, Computation, and Statistical Inference. Journal of Econometrics, (77), 87-103.

Kaplan, G., Goldberg, D., Everson, S. et al. (1996). Perceived Health Status and Morbidity and Mortality: Evidence from the Kuopio Ischaemic Heart Disease Risk Factor Study. Int. J. Epidemiol, (25), 259-65.

Kaplan, G., Pamuk, E., Lynch, J., Cohen, R. \& Balfour, J. (1996). Inequality in income and Mortality in the United States: Analysis of Mortality and Potential Pathways. BMJ (Clinical Research ed.), 312(7037), 999-1003.

Kawachi, I., Kennedy, B. \& Glass, R. (1999). Social Capital and Self-Rated Health: A Contextual Analysis. American Journal of Public Health, 89(8), 1187-1193.

Kawachi, I., Kennedy, B., Lochner, K. \& Prothrow-Stith, D. (1997). Social Capital, Income Inequality, and Mortality. American Journal of Public Health, 87(9), 1491-1498.

Kennedy, B., Kawachi, I., Glass, R. \& Prothrow-Stith, D. (1998). Income Distribution, Socioeconomic Status, and Self Rated Health in the United States: Multilevel Analysis. BMJ (Clinical Research, ed.), 317(7163), 917-921.

Kennedy, B., Kawachi, I. \& D. ProthrowStith (1996). Income Distribution and Mortality: Cross Sectional Ecological Study of the Robin Hood Index in the United States. BMJ, (312), 1004-1007.

Kolenikov, S. \& Angeles, G. (2009). Socioeconomic Status Measurement with Discrete Proxy Variables: Is Principal Component Analysis a Reliable Answer? Review of Income and Wealth, 55(1), 128-165. 
LeClere, F. \& Soobader, M. (2000). The Effect of Income Inequality on the Health of Selected U.S. Demographic Groups. American Journal of Public Health, 90(12), 1892-1897.

Leeuw, J. \& Meijer, E. (Eds.). (2008). Handbook of Multilevel Analysis. Nueva York, NY: Springer New York.

Lutter, R. \& Morrall, J. (1994). Health-Health Analysis: A New Way to Evaluate Health and Safety Regulation. Journal of Risk and Uncertainty, 8(1), 43-66.

Lynch, J., Davey-Smith, G., Kaplan, G. \& House, J. (2000). Income Inequality and Mortality: Importance to Health of Individual Income, Psychosocial Environment, or Material Conditions. BMJ (Clinical Research ed.), 320(7243), 1200-1204.

Lynch, J., Kaplan, G. \& Pamuk, E. (1998). Income Inequality and Mortality in Metropolitan Areas of the United States. American Journal of Public Health, (88), 1074-1080.

Macintyre, S., \& Ellaway, A. (2000). Neighbourhood Cohesion and Health in Socially Contrasting Neighbourhoods: Implications for the Social Exclusion and Public Health Agendas. Health Bulletin, 58(6), 450-456.

Marmot, M., Rose, G., Shipley, M. \& Hamilton, P. (1978). Employment Grade and Coronary Heart Disease in British Civil Servants. Journal of Epidemiology and Community Health, (32), 244-249.

Mellor, J. \& Milyo, J. (1999). Income Inequality and Individual Health: Evidence from the Current Population Survey. Boston: Robert Wood Johnson Health Policy Scholars, Boston University School of Management, Working Paper, 8.

MESEP, DANE-DNP. (2010). Misión para el empalme de las series de empleo, pobreza y desigualdad (Mesep). Bogotá: Departamento Nacional de Planeación.

Mullahy, J., Robert, S. \& Wolfe, B. (2001). Health, Income, and Inequality: Review and Redirection for the Wisconsin Russell Sage Working Group. En Russell Sage Foundation, Working Paper 1-32.

Organización Mundial de la Salud (OMS) (2011). Cerrando la brecha: la política de acción sobre los determinantes sociales de la salud, Conferencia mundial sobre los determinantes sociales de la salud. Río de Janeiro, 19-21 de octubre.

Poel, E. van de \& Hosseinpoor, N. (2008). Socioeconomic Inequality in Malnutrition in Developing Countries. Bulletin of the World Health Organization, 86(4), 241-30. 
PNUD. (2010). Informe regional sobre desarrollo humano para América Latina y el Caribe 2010: Actuar sobre el futuro: romper la transmisión intergeneracional de la desigualdad. PNUD.

PNUd. (1991-2009). Colombia. Desarrollo humano. Bogotá: Informes de Desarrollo Humano, PNUD.

Rodgers, G. (1979). Income and Inequality as Determinants of Mortality : An International Cross-Section Analysis. Population, 33(2), 343-351.

Ruiz, F. (2011). Health Equity in the Colombian Health System: Effects from a Health Reform Implementation. Toronto: Presentación iHea 2011.

Sapolski, R. (1993). Endocrinology Alfresco: Psychoendocrine Studies of Wild Baboons. Recent Progress in Hormone Research, (48), 437-468.

Smith, J. (2004). Unraveling the SES-Health Connection. En Waite, L. (Ed.). Aging, Health and Public Policy: Demographic and Economic Perspectives. Population and Development Review. Nueva York: Population Council, suplemento del vol. 30.

Smith, J. (1999). Healthy Bodies and Thick Wallets: The Dual Relation between Health and Economic Status. Journal of Economic Perspectives, 13(2), 145-166.

Soobader, M. \& LeClere, F. (1999). Aggregation and the Measurement of Income Inequality: Effects on Morbidity. Social Science \& Medicine, 48(6), 733-744.

Subramanian, S. \& Kawachi, I. (2004). Income Inequality and Health: What Have We Learned so Far? Epidemiologic Reviews, (26), 78-91.

Subramanian, S., Kim, D. \& Kawachi, I. (2002). Social Trust and Self-Rated Health in US Communities: A Multilevel Analysis. Journal of Urban Health: Bulletin of the New York Academy of Medicine, 79(4, suplemento 1), S21-34.

Subramania, S., Kawachi, I. \& Kennedy, B. (2001). Does the State you Live in Make a Difference? Multilevel Analysis of Self-Rated Health in the US. Social Science \& Medicine, 53(1), 9-19.

Veenstra, G. (2005). Location, Location, Location: Contextual and Compositional Health Effects of Social Capital in British Columbia, Canada. Social Science \& Medicine, 60(9), 2059-2071.

Viscusi, K. (1994). Risk-Risk Analysis. Journal of Risk and Uncertainty, 8(1), 5-17. 
Wagstaff, A. \& Doorslaer, E. van. (2000a). Income Inequality and Health: What Does the Literature Tell Us? Annual review of Public Health, 21(25), 543-567.

Wagstaff, A. \& Doorslaer, E. van. (2000b). Equity in Health Care Finance and Delivery. En Culyer, A. \& Newhouse, J. (Eds.). Handbook of Health Economics. Oxford: Elsevier.

Waitzman, N. \& Smith, K. (1998b). Separate but Lethal: The Effects of Economic Segregation on Mortality in Metropolitan America. Milbank Quarterly, 76(3), 341-373.

Waldmann, R. (1992). Income Distribution and Infant Mortality. Quarterly Journal of Economics, 107(4), 1283-1302.

Wildman, J. (2003). Modelling Health, Income and Income Inequality: The Impact of Income Inequality on Health and Health Inequality. Journal of Health Economics, (22), 521-538.

Wildman, J. (2001). The Impact of Income Inequality on Individual and societal Health: Absolute Income, Relative Income and Statistical Artefacts. Health Economics, (10), 357-361.

Wilkinson, R. (1998). Low Relative Income Affects Mortality. British Medical Journal, $316(7144), 1611$.

Wilkinson, R. (1997). Health Inequalities: Relative or Absolute Material Standards? British Medical Journal, 314(7080), 591-595.

Wilkinson, R. (1996). Unhealthy Societies: The afflictions of inequality. Londres: Routledge/Kegan Paul.

Wilkinson, R. (1992). Income Distribution and Life-Expectancy. British Medical Journal, (304), 165-168.

Wilkinson, R \& Pickett, K. (2009). Income Inequality and Social Dysfunction. Annual Review of Sociology, 35(1), 493-511.

Wilkinson, R. \& Pickett, K. (2006). Income Inequality and Population Health: A Review and Explanation of the Evidence. Social Science \& Medicine, 62(7), 1768-1784.

Recibido el 28 de julio de 2014. Aceptado el 21 de agosto de 2015. 